\title{
Sex-inducing effect of a hydrophilic fraction on reproductive switching in the planarian Dugesia ryukyuensis (Seriata, Tricladida)
}

Kazuya Kobayashi ${ }^{1 *}$ and Motonori Hoshi ${ }^{2}$

\begin{abstract}
Background: The mechanisms underlying the switching from an asexual to a sexual mode of reproduction, and vice versa, remain unknown in metazoans. In planarians, asexual worms acquire cryptic sexuality when fed with sexual worms, indicating that sexual worms contain a sex-inducing substance. Although such a chemical compound will provide clues about the mechanisms underlying the switching, information on the sex-inducing substance is poor. In order to identify this substance, we have established an assay system for sexual induction in asexual worms of Dugesia ryukyuensis by feeding them with sexual worms. Here, we carried out an isolation study on the sex-inducing substance using this assay system.

Results: After centrifugation of sexual worms homogenised in saline solution, we found that not only did the precipitate have a sex-inducing effect on the asexual worms, which has been shown previously, but the cytosolic fraction did as well. We confirmed that the sex-inducing activity in the cytosolic fraction was recovered in a hydrophilic fraction separated on an octadecylsilane (ODS) column. We showed that the sex-inducing substance in the hydrophilic fraction is papain-resistant and a putative low-molecular-weight compound of less than 500. We also suggest the presence of an enhancer of sexual induction with a molecular weight (MW) of more than $5 \mathrm{~K}$ in the hydrophilic fraction.

Conclusion: Our experiments showed the existence of a sex-inducing substance and an enhancer of sex-induction in a hydrophilic fraction, and a putative hydrophobic sex-inducing substance in the precipitate. Sexual induction in the asexual worms might be triggered by additive or synergistic effects of these chemical compounds.
\end{abstract}

Keywords: Reproductive strategy, asexual reproduction, sexual reproduction, sex-inducing substance, Dugesia ryukyuensis, planarians

\section{Background}

Many metazoans can reproduce sexually as well as asexually. When environmental factors are favourable, asexual reproduction is employed to exploit suitable conditions for survival. In general, they switch from an asexual to a sexual mode of reproduction, when individual survival is greatly hampered. Variations found in the offspring resulting from sexual reproduction allow some individuals to be better suited for survival and provide a mechanism for selective adaptation. Therefore,

\footnotetext{
* Correspondence: kobayashikyram@a5.keio.jp

'Center for Integrated Medical Research, School of Medicine, Keio University, 35 Shinjuku-ku, Tokyo, 160-8582, Japan

Full list of author information is available at the end of the article
}

the reproductive strategy of switching between asexual and sexual reproduction may contribute to fitness. However, the mechanisms underlying the switch between the 2 modes remain unknown.

Some freshwater planarians (Platyhelminthes, Turbellaria, Seriata, Tricladida) reproduce asexually as well as sexually [1-6]. The sexual worms are hermaphrodites, while the asexual worms reproduce by fission without forming any sexual organs. Certain worms develop sexual organs during the colder season, while they reproduce asexually with much degenerated gonads during the warmer season of the year. Asexual worms can switch to the sexual mode if they are fed sexual worms; this means that the sexual worms contain a sex-

\section{C) Biomed Central}


inducing substance that is not species-specific $[7,8]$. The putative sex-inducing substance is likely to provide clues about the mechanisms underlying the switch from an asexual to a sexual mode of reproduction. However, very little information is available on the sex-inducing substance. Grasso et al. [9] tried to purify a sex-inducing substance contained in Polycelis nigra by using asexual worms of Dugesia gonocephala as test worms. They homogenised $P$. nigra in saline solution, and then 3 fractions of the precipitate and 1 fraction of the supernatant were obtained after a 3-step centrifugation. Sex-inducing activity was detected only in the first and second precipitates but not in the supernatant (the cytosolic fraction). This suggested that the sex-inducing substance contained in $P$. nigra might be a hydrophobic compound.

In order to isolate and identify this sex-inducing substance, we have established a bioassay system for sexual induction. We planned to use asexual worms exclusively as the test worms for isolating and identifying the sexinducing substance. Other important issues regarding the animals were the supply (abundance, constancy, ease, and readiness), easy culture in laboratory, resistance to operations such as ablation, and adequate body size. Considering all these points, we chose the $\mathrm{OH}$ strain of Dugesia ryukyuensis as the test worms because spontaneous sexual induction has never been observed in this strain under laboratory conditions. No functional sexual organs were externally recognised in the test worms before or after they consumed conspecific asexual worms and chicken liver - their daily food. However, when they were fed the minced worms of B. brunnea, an oviparous species, they developed hermaphroditic sexual organs. After an intensive and extensive survey of the appropriate conditions of the bioassay, such as the population density, feeding procedure, and temperature, we established a simple, reliable, and a relatively quick assay system [10-14]. We divided the process of sexual induction into 5 distinct stages by morphological changes [[10,12], Additional file 1]. In stage 1, the ovaries became sufficiently large to be externally apparent behind the head, yet no oocytes or other sexual organs were detectable. In stage 2, oocytes appeared in the ovaries, but other sexual organs remained undetectable. In stage 3 , the primordial testes emerged and a copulatory apparatus became visible as a white speck in the post-pharyngeal region. In stage 4, yolk gland primordia developed, and spermatocytes appeared in the testes. In stage 5, matured yolk glands formed, and many matured spermatozoa were detectable in the testes. We also found that sexual induction has a point-of-no-return between stages 2 and 3 . The test worms at stages 1 and 2 return to being asexual if feeding on $B$. brunnea is stopped. On the contrary, the test worms at stages 3 onward keep developing sexual organs, even though feeding on B. brunnea is stopped. Briefly, by external observation, we can recognise the test worms with only a pair of ovaries and those with a copulatory apparatus as the state before and after the point-of-noreturn, respectively. Furthermore, Dryg is expressed from the primordial to the mature yolk glands at stages 3 onward, which means that it can be used as a marker gene for the degree of sexual induction after the pointof-no-return [15].

In the present study, we used sexual worms of D. ryukyuensis as a source of the sex-inducing substance. Sexinducing activity was examined by external observation and Dryg expression. After centrifugation, we found that both the cytosolic fraction and the precipitate displayed sex-inducing activity in the asexual worms. Furthermore, we recovered and analysed the sex-inducing activity in a more hydrophilic fraction (fraction $\mathrm{M} 0+\mathrm{M} 10$ ) by using a commercial octadecylsilane (ODS) column. We have not been able to establish if more than 1 substance is responsible for sexual induction, but until this is resolved, we will refer to the substance in singular.

\section{Results}

Sex-inducing activity in the precipitates from samples extracted with phosphate-buffered saline

Because the test worms avoided consuming minced sexual worms of the $\mathrm{OH}$ strain, we could not estimate the specific activity (purification) of the sex-inducing substance in the sexual worms of D. ryukyuensis by using the established purification procedure (Figure 1). To estimate the specific activity, we instead used B. brunnea as a source of the sex-inducing substance. About $400 \mathrm{mg}$ of B. brunnea was sufficient to induce the sexual mode of reproduction in 25 test worms, when the test worms were fed with minced worms of B. brunnea (Table 1). Following Grasso et al. [9], we homogenised $4 \mathrm{~g}$ of worms in phosphate-buffered saline (PBS) and then obtained 2 fractions of the precipitates after a 2-step centrifugation (Figure 1). Most test worms developed a pair of ovaries, but no copulatory apparatus, when they were fed about $400 \mathrm{mg}$ of these precipitates (Table 1). This suggested that even after ultracentrifugation, the sex-inducing substance was still contained in the cytosolic fraction.

\section{Sex-inducing activity of extracts fractioned on the ODS column}

The cytosolic fraction was desalted and separated on a commercial octadecylsilane (ODS) column (Figure 1). We could not confirm whether the flow-through fraction contained a sex-inducing substance, because the test worms could not eat it due to a high salt concentration. Strong sex-inducing activity was recovered in the fractions eluted with water and 10\% methanol (M0 and M10, respectively; Figure 2 and Table 2). This indicates 


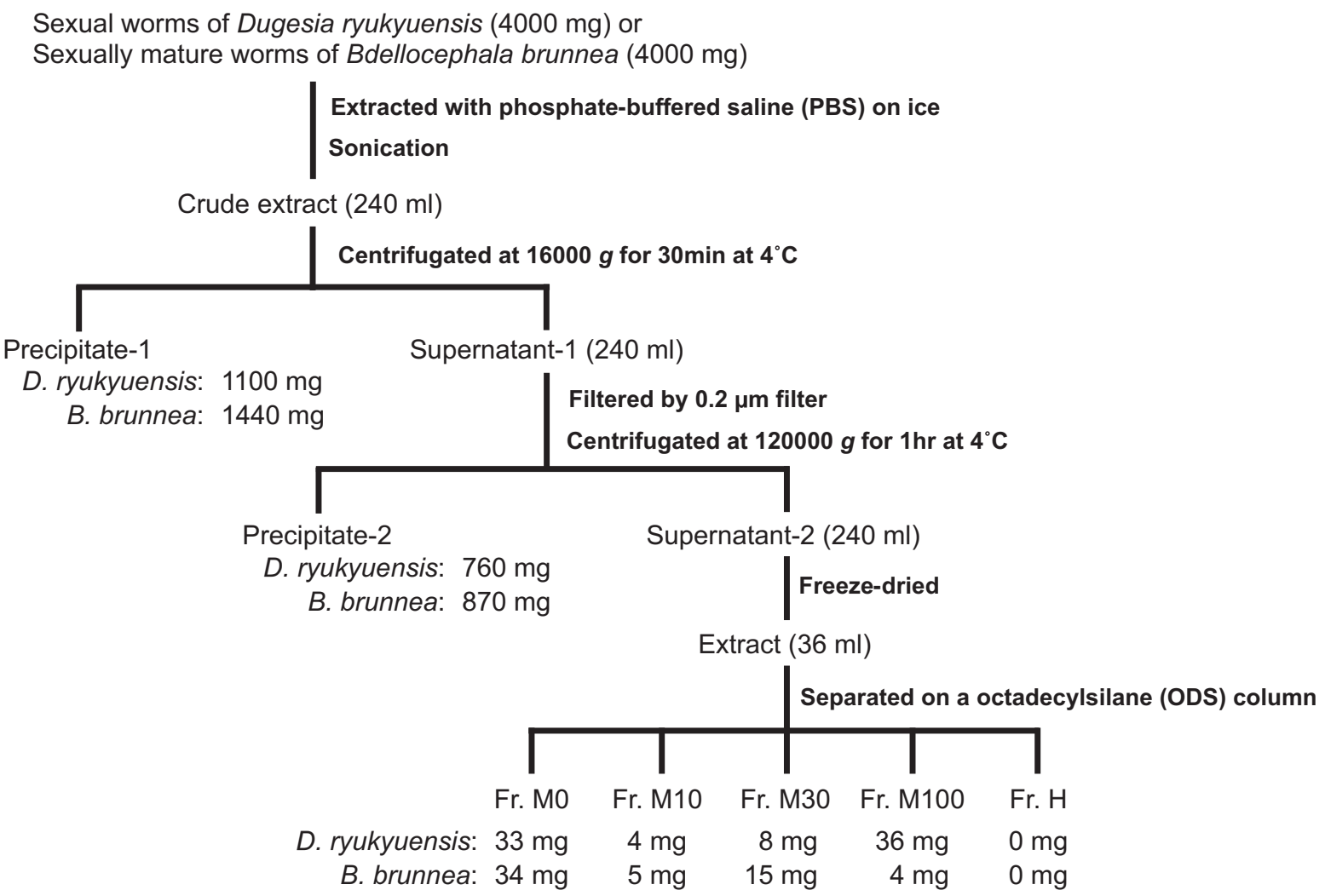

Figure 1 Procedure for the fractionation of Dugesia ryukyuensis and Bdellocephala brunnea. See the text for experimental details. Fr., fraction.

that sexual planarians have a hydrophilic sex-inducing substance. The weight of the M0 and M10 fractions was almost the same between the sexual worms of $D$. ryukyuensis and B. brunnea (Figure 1). The sex-inducing activity of the M0 and M10 fractions was expressed as relative values of Dryg expression based on minced worms of B. brunnea (400 mg; Figure 3). In the sexual worms, the sex-inducing activity per weight of these fractions was 2.3- and 6.6-fold higher than that of $B$. brunnea, respectively. This means that the quality, quantity, or both of the sex-inducing substance in $D$. ryukyuensis differs from the substance in B. brunnea. The $\mathrm{M} 0$ and M10 fractions were derived from $4 \mathrm{~g}$ of $B$. brunnea. Because 25 test worms cannot finish eating $4 \mathrm{~g}$ on this assay schedule, the sex-inducing activity of $4 \mathrm{~g}$ of B. brunnea was extrapolated from that based on consumption of $400 \mathrm{mg}$. The sex-inducing activity was increased approximately 3 - and 7-fold in the M0 and

Table 1 Sex-inducing activity of the precipitates from samples extracted with phosphate-buffered saline (PBS)

\begin{tabular}{lccc}
\hline Test food & & $\begin{array}{c}\text { Number of test worms that developed a pair of } \\
\text { ovaries (\%) }\end{array}$ & $\begin{array}{c}\text { Number of test worms that developed a copulatory } \\
\text { apparatus (\%) }\end{array}$ \\
\hline $\begin{array}{l}\text { Dugesia } \\
\text { ryukyuensis }\end{array}$ & ppt-1 & $44 / 49(89.8 \pm 2.3)$ & $1 / 49(2.0 \pm 2.0)$ \\
& ppt-2 & $21 / 50(42.0 \pm 6.0)$ & $0 / 50(0.0 \pm 0.0)$ \\
$\begin{array}{l}\text { Bdellocephala } \\
\text { brunnea }\end{array}$ & ppt-1 & $36 / 50(72.0 \pm 12.0)$ & $0 / 50(0.0 \pm 0.0)$ \\
& ppt-2 & $4 / 49(8.1 \pm 3.9)$ & $0 / 49(0.0 \pm 0.0)$ \\
& $\begin{array}{l}\text { Minced } \\
\text { worms }\end{array}$ & $49 / 49(100.0 \pm 0.0)$ & $47 / 49(96.0 \pm 4.0)$ \\
\hline
\end{tabular}

The precipitates were obtained as described in Figure 1. Twenty-five test worms were fed approximately $400 \mathrm{mg}$ of each sample for 4 weeks. Means and standard errors of duplicated experiments are shown. ppt, precipitate. 


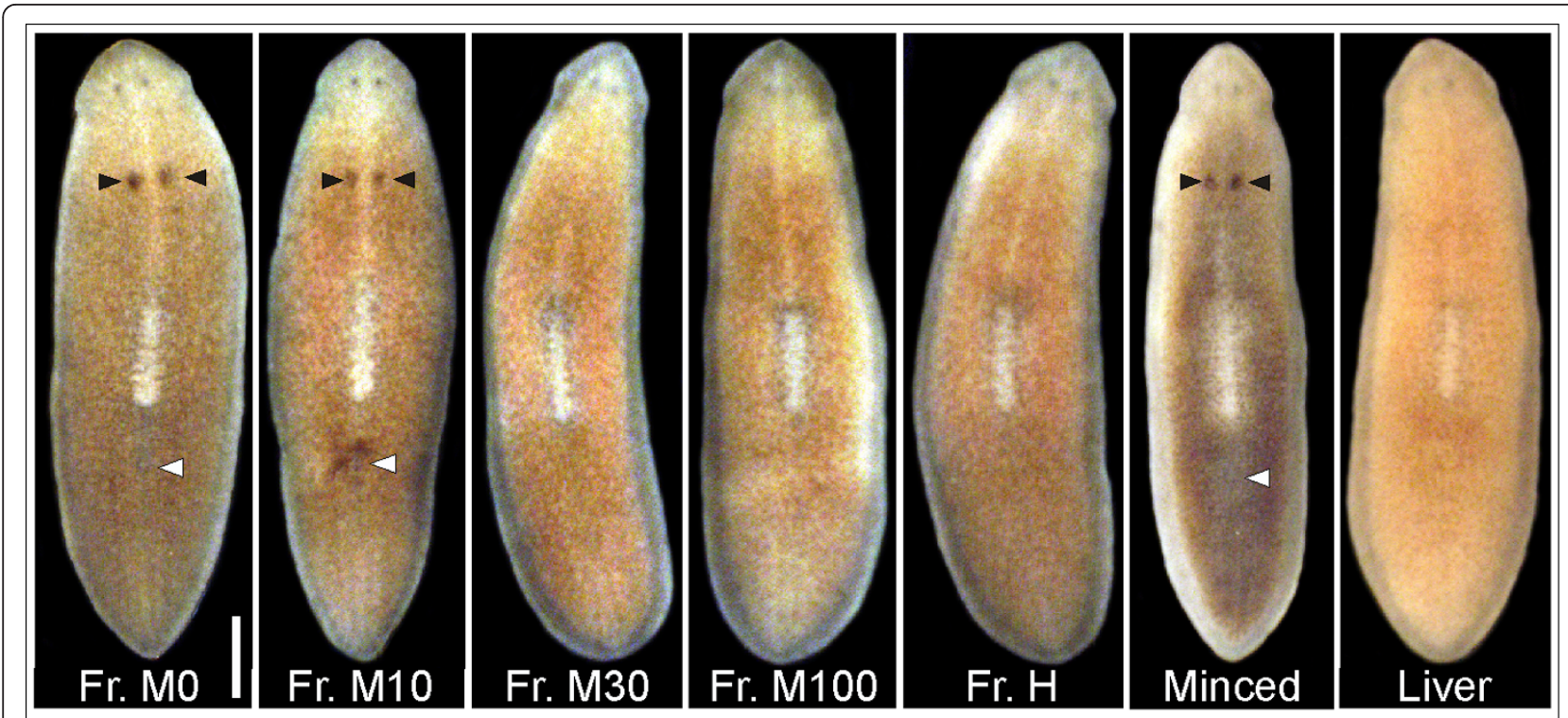

Figure 2 External sexual appearances of test worms fed Dugesia ryukyuensis extracts as shown in Table 2. Ventral views of test worms at the fourth week of feeding are shown. Test worms were fed fractions M0 (Fr. M0), M10 (Fr. M10), M30 (Fr. M30), M100 (Fr. M100), H (Fr. H), minced Bdellocephala brunnea (Minced), and chicken liver (Liver). Arrowheads represent an ovary pair (black) and a copulatory apparatus (white). The images are arranged with the anterior to the top. Scale bars represent $2 \mathrm{~mm}$.

M10 fractions, respectively, using the purification procedure established (Table 3).

\section{Analysis of a sex-inducing effect of the $M 0+M 10$ fraction}

To determine the effective concentration of the $\mathrm{M} 0+$ M10 fraction for sexual induction, we carried out a dilution experiment (Table 4). Dilutions of 5-fold or higher cannot induce a sexual mode of reproduction in the test worms, but even a 25 -fold dilution could cause development of a pair of ovaries in the test worms. Based on this, we set the minimum feeding conditions for the subsequent experiments at $4 \mathrm{~g}$ of sexual worms for 25 test animals for 4 weeks.

Sakurai [8] reported that the asexual worms of $D$. japonica became sexual and developed several

Table 2 Sex-inducing activity of the extracts separated on a commercial octadecylsilane (ODS) column

\begin{tabular}{|c|c|c|c|}
\hline \multicolumn{2}{|c|}{ Test food } & \multirow{2}{*}{$\begin{array}{c}\text { Number of test worms that developed a pair of } \\
\text { ovaries (\%) } \\
50 / 50(100.0 \pm 0.0)\end{array}$} & \multirow{2}{*}{$\begin{array}{c}\text { Number of test worms that developed a copulatory } \\
\text { apparatus (\%) }\end{array}$} \\
\hline Dugesia & Fr. MO & & \\
\hline & Fr. M10 & $47 / 49(96.0 \pm 4.0)$ & $44 / 49(89.5 \pm 6.5)$ \\
\hline & Fr. M30 & $0 / 48(0.0 \pm 0.0)$ & $0 / 48(0.0 \pm 0.0)$ \\
\hline & Fr. M100 & $0 / 48(0.0 \pm 0.0)$ & $0 / 48(0.0 \pm 0.0)$ \\
\hline & Fr. $\mathrm{H}$ & $0 / 50(0.0 \pm 0.0)$ & $0 / 50(0.0 \pm 0.0)$ \\
\hline \multirow{6}{*}{$\begin{array}{c}\text { Bdelloceplala } \\
\text { brunnea }\end{array}$} & Fr. MO & $50 / 50(100.0 \pm 0.0)$ & $44 / 50(88.0 \pm 8.0)$ \\
\hline & Fr. M10 & $45 / 49(91.8 \pm 4.3)$ & $39 / 49(79.6 \pm 4.5)$ \\
\hline & Fr. M30 & $0 / 50(0.0 \pm 0.0)$ & $0 / 50(0.0 \pm 0.0)$ \\
\hline & Fr. M100 & $0 / 48(0.0 \pm 0.0)$ & $0 / 48(0.0 \pm 0.0)$ \\
\hline & Fr. $H$ & $0 / 50(0.0 \pm 0.0)$ & $0 / 50(0.0 \pm 0.0)$ \\
\hline & $\begin{array}{l}\text { Minced } \\
\text { worms }\end{array}$ & $49 / 49(100.0 \pm 0.0)$ & $47 / 49(96.0 \pm 4.0)$ \\
\hline Chicken liver & & $0 / 50(0.0 \pm 0.0)$ & $0 / 50(0.0 \pm 0.0)$ \\
\hline
\end{tabular}

The extracts were obtained as described in Figure 1. See Materials and methods for test food. Twenty-five test worms were also fed approximately 400 mg of minced worms and chicken liver, respectively, for 4 weeks. Means and standard errors of duplicated experiments are shown. Fr., fraction. 


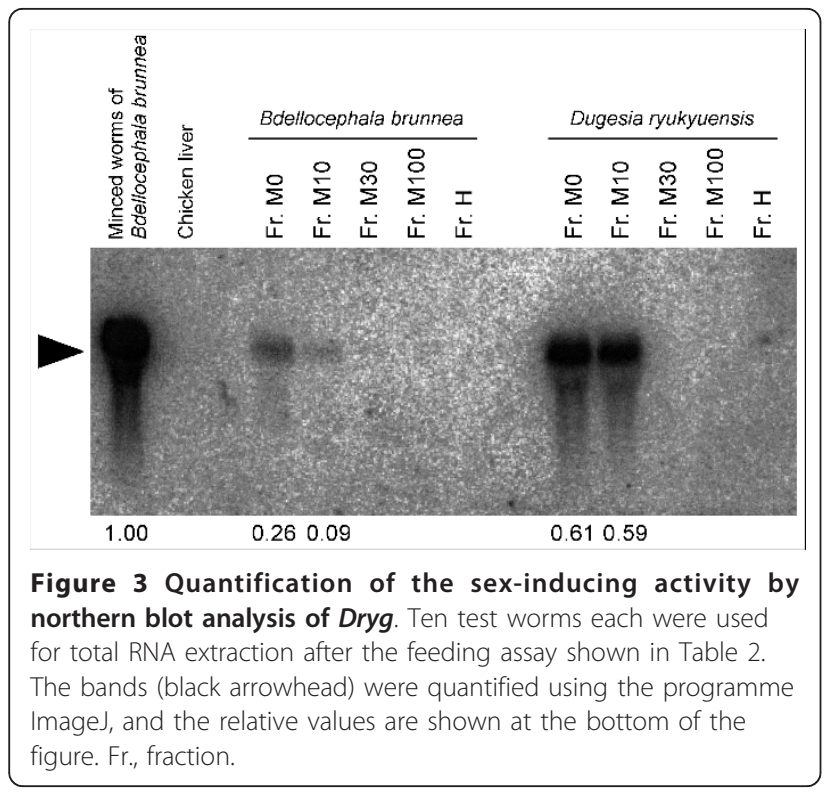

supernumerary ovary pairs when fed with $B$. brunnea. We have showed that in the test worms with acquired sexuality, many supernumerary ovary pairs were apparently induced by feeding them with $B$. brunnea (Additional file 2). Supernumerary ovaries were also induced in the test worms with acquired sexuality by feeding them the M0 + M10 fraction of conspecific sexual worms (Figure 4). Sakurai [8] also reported that the feeding of the head portion of B. brunnea did not cause complete sexual induction of asexual worms, whereas that of the portions other than the head did cause complete sexual induction. Following Sakurai [8], we fed the test worms the M0 + M10 fractions derived from 3 fragments of conspecific sexual worms (Figure 5). The M0 + M10 fractions from the middle (M) and tail (T) fragments were effective for ovarian induction, but those from the head $(\mathrm{H})$ fragments were ineffective (Table 5). Approximately $70 \%$ of the test worms acquired full sexuality after consuming the M0 + M10 fractions from the $\mathrm{M}$ and $\mathrm{T}$ fragments, whereas no test worms acquired sexuality by feeding on the M0 + M10 fraction from the $\mathrm{H}$ fragments (Table 5).

When the homogenate of the sexual worms was boiled at $100^{\circ} \mathrm{C}$ for $15 \mathrm{~min}$, the sex-inducing activity in the M0 + M10 fraction decreased sharply, and the test worms only developed a pair of ovaries (data not shown). To confirm whether the sex-inducing substance contained in the M0 + M10 fraction was a protein, we used a cysteine protease, papain, and ultrafiltration. The procedure used to separate the M0 + M10 fraction is shown in Figure 6. First, we separated the M0 + M10 fraction by ultrafiltration through $5-\mathrm{K}$ molecular weight cut-off (MWCO) membranes, which remove papain (molecular weight [MW]: $22 \mathrm{kD}$ ). Sex-inducing activity was only recovered in the fraction of less than $5 \mathrm{~K} \mathrm{MW}(<5-\mathrm{K}$ MW fraction) (Table 6). Interestingly, evaluation of sexinducing activity on the basis of Dryg expression revealed that the $<5-\mathrm{K} M W$ fraction showed a 5.6-fold increase in sex-inducing activity by the addition of the > $5-\mathrm{K} \mathrm{MW}$ fraction, even though the $>5-\mathrm{K} \mathrm{MW}$ fraction alone did not have any sex-inducing activity (Figure 7). This suggests the existence of an enhancer for sexual induction in the $>5-\mathrm{K} \mathrm{MW}$ fraction. Next, we performed protein digestion with papain for the M0 + M10 fraction. The test worms not only developed a pair of ovaries but also a copulatory apparatus when fed the protein-digested < 5-K MW fraction (Table 6), and the sex-inducing activity increased 1.7-fold (Figure 7). Finally, the $<5-\mathrm{K} \mathrm{MW}$ fraction after papain treatment was dialysed using approximately $30 \mathrm{~L}$ water for $48 \mathrm{~h}$. The otherwise $<5-\mathrm{K}$ MW fraction after papain treatment was placed under the same conditions, except for dialysis, as a control to consider the possibility that the sex-inducing substance is non-specifically degraded (Figure 7 and Table 6). The test worms did not attain complete sexuality after being fed the retentate after dialysis of $500 \mathrm{MWCO}$, whereas they became sexual when fed the control food (Figure 7 and Table 6). This suggested that the hydrophilic sex-inducing substance is a lowMW compound (MW: < 500). The dialysate should contain the sex-inducing substance. However, we did not examine the dialysate because concentrating the large amount of dialysate (approximately $30 \mathrm{~L}$ ) was not realistic in terms of an isolation study (effective recovery of the sex-inducing substance).

\section{Discussion}

The existence of a sex-inducing substance was demonstrated by Grasso and Benazzi [7]. Grasso et al. [9] reported that sex-inducing activity was recovered in the

Table 3 Purification of a sex-inducing substance in the fractions M0 and M10

\begin{tabular}{lllll}
\hline Samples & Total weight $(\mathbf{m g})$ & Sex-inducing activity & Specific activity & Purification (fold) \\
\hline Minced worms & 4000 & 10 & 0.0025 & $1 \times$ \\
Fr. M0 & 34 & 0.258 & 0.0076 & $3 \times$ \\
Fr. M10 & 5 & 0.088 & 0.0176 & $7 \times$ \\
\hline
\end{tabular}

Sex-inducing activity was represented as relative values of the expression of Dryg based on minced worms of Bdellocephala brunnea (400 mg) in Figure 3. Fr. fraction. 
Table 4 Dilution assay of sex-inducing activity in the M0 + M10 fraction of Dugesia ryukyuensis

\begin{tabular}{ccc}
\hline $\begin{array}{c}\text { Dilution } \\
\text { series }\end{array}$ & $\begin{array}{c}\text { Number of test worms that developed a pair of ovaries } \\
(\%)\end{array}$ & $\begin{array}{c}\text { Number of test worms that developed a copulatory apparatus } \\
(\%)\end{array}$ \\
\hline 1 & $45 / 50(90.0 \pm 6.0)$ & $38 / 50(76.0 \pm 16.0)$ \\
$1 / 2$ & $40 / 50(80.0 \pm 4.0)$ & $14 / 50(28.0 \pm 4.0)$ \\
$1 / 5$ & $35 / 49(71.5 \pm 3.5)$ & $0 / 49(0.0 \pm 0.0)$ \\
$1 / 10$ & $33 / 50(62.0 \pm 2.0)$ & $0 / 50(0.0 \pm 0.0)$ \\
$1 / 25$ & $3 / 50(6.0 \pm 6.0)$ & $0 / 50(0.0 \pm 0.0)$ \\
$1 / 125$ & $0 / 48(0.0 \pm 0.0)$ & $0 / 48(0.0 \pm 0.0)$ \\
$1 / 625$ & $0 / 50(0.0 \pm 0.0)$ & $0 / 50(0.0 \pm 0.0)$ \\
\hline
\end{tabular}

To prepare test food, each dilution sample was mixed with $150 \mu$ l of chicken liver homogenate and then freeze-dried. Twenty-five test worms were fed for 4 weeks. Means and standard errors of duplicated experiments are shown.

precipitate, but not in the cytosolic fraction, obtained after centrifugation of the homogenate of sexual worms in saline solution. Since then, there has been no substantial improvement in the isolation of a sex-inducing substance from sexual worms. We tried to purify the sex-inducing substance contained in D. ryukyuensis by using conspecific sexual worms. Following Grasso et al. [9], we homogenised $4 \mathrm{~g}$ of sexual worms (about 320 worms) in phosphate-buffered saline (PBS) and then obtained 2 fractions of the precipitate after 2-step centrifugation (Figure 1). The test worms did not attain complete sexuality when fed precipitates 1 and 2 , although they did develop a pair of ovaries (Table 1). Similarly, feeding the test worms with $\sim 400 \mathrm{mg}$ of $B$. brunnea precipitates also resulted in the development of a pair of ovaries only. Because $\sim 400 \mathrm{mg}$ of minced worms of B. brunnea was sufficient to induce a sexual mode of reproduction in 25 test worms, it was expected

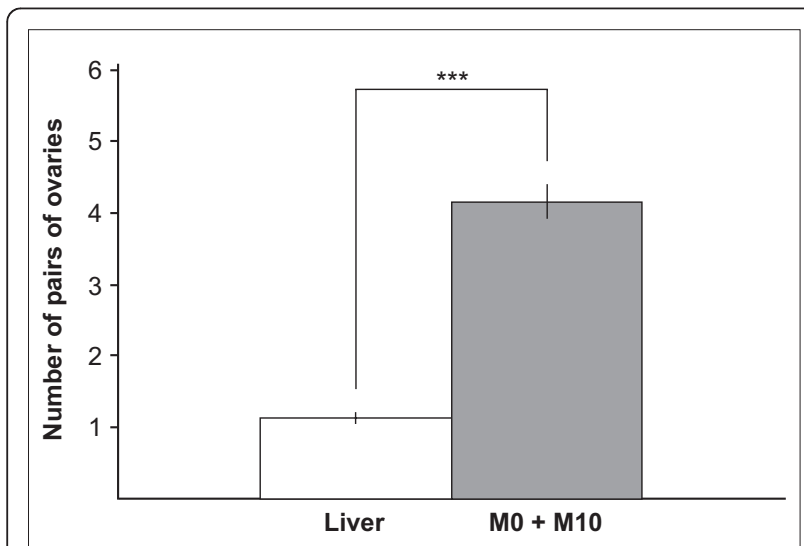

Figure 4 Induction of supernumerary ovary pairs by feeding with the M0 + M10 fraction. Test worms with acquired sexuality induced significantly more supernumerary ovary pairs by feeding with the $\mathrm{M0}+\mathrm{M} 10$ fraction than that with chicken liver for 2 weeks (Welch's t-test: $\left.n_{M 0}+M_{10}=25, n_{\text {Liver }}=25 ; P=1.59 E-12\right)$. Error bars represent the standard error. The M0 + M10 fraction of Dugesia ryukyuensis was obtained as described in Figure 6 . To prepare test food, the M0 + M10 fraction was mixed with $150 \mu \mathrm{l}$ of chicken liver homogenate and then freeze-dried. that the cytosolic fraction contained the sex-inducing substance. In P. nigra, sex-inducing activity was recovered only in the precipitate [9], and the responsible substance might be a hydrophobic compound. In order to confirm whether the sex-inducing substance in the cytosolic fraction of D. ryukyuensis is hydrophobic or hydrophilic, we separated the cytosolic fraction by using a commercial octadecylsilane (ODS) column. Strong sexinducing activity was recovered in the fractions eluted with water and $10 \%$ methanol (fractions M0 and M10, respectively) (Figures 2 and 3, Table 2). This means that

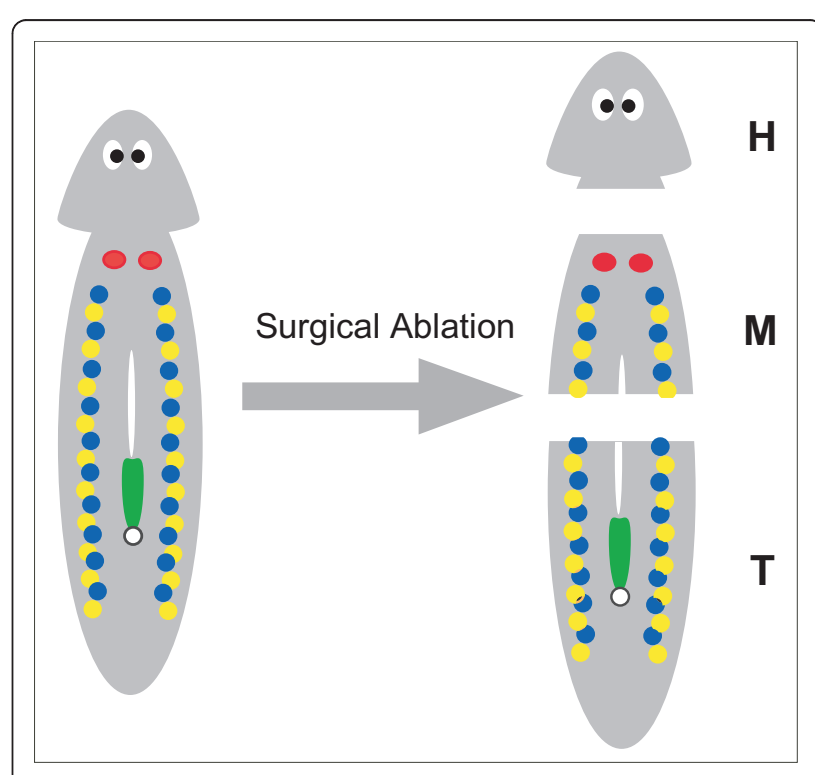

Figure $\mathbf{5}$ Scheme of the surgical ablation of sexual worms. By surgical ablation, 3 different fragments were obtained with respect to the topological position of the sexual organs: the head $(\mathrm{H})$ fragment had no sexual organs; the middle (M) fragment had a pair of ovaries, testes, and yolk glands; the tail (T) fragment had testes, yolk glands, and a copulatory apparatus. Coloured regions correspond with the sexual organs: red, ovary; blue, testis; yellow, yolk gland; green, a copulatory apparatus with a genital pore. The white region in the body is the pharynx. Figure 2 in Kobayashi et al [13] was modified. 
Table 5 Sex-inducing activity in 3 fragments obtained according to the topological position of the sexual organs

\begin{tabular}{lcc}
\hline Test food & $\begin{array}{c}\text { Number of test worms that developed a pair of } \\
\text { ovaries (\%) }\end{array}$ & $\begin{array}{c}\text { Number of test worms that differentiated a copulatory } \\
\text { apparatus (\%) }\end{array}$ \\
\hline $\begin{array}{c}\text { Head fragments } \\
\text { Middle }\end{array}$ & $0 / 25(0.0)$ & $0 / 25(0.0)$ \\
fragments & $24 / 25(96.0)$ & $18 / 25(72.0)$ \\
Tail fragments & $24 / 25(96.0)$ & $15 / 25(60.0)$ \\
\hline
\end{tabular}

The different fragments are described in Figure 5.

the sex-inducing substance contained in D. ryukyuensis is a hydrophilic as well as a hydrophobic compound.

Although sex-inducing activity in the M0 + M10 fraction was extremely decreased when the homogenate of the sexual worms was boiled at $100^{\circ} \mathrm{C}$ for $15 \mathrm{~min}$, the test worms did develop a pair of ovaries (data not shown). In addition, although a 2 -fold diluted $\mathrm{M} 0+$ M10 fraction was sufficient to induce a sexual mode of reproduction in the test worms, even a 25 -fold dilution could cause development of a pair of ovaries in the test worms (Table 4). This suggests that the M0 + M10 fraction contains an ovary-inducing substance that can operate partially independently of other substances involved in acquiring complete sexuality. We found a seasonal change in the quality, quantity, or both, of the sex-inducing substance contained in B. brunnea [14]. When $B$. brunnea were immature in summer, the sexinducing activity of the test worms was extremely low. The feeding with $B$. brunnea frozen in summer induced merely a pair of ovaries in spite of the long-term feeding. On the contrary, when they were sexually mature in winter, the sex-inducing activity was higher. This implied the existence of the ovary-inducing substance. Conversely, the test worms with acquired sexuality developed many supernumerary ovary pairs after consuming B. brunnea and the M0 + M10 fraction of conspecific sexual worms (Figure 4, Additional file 2). The

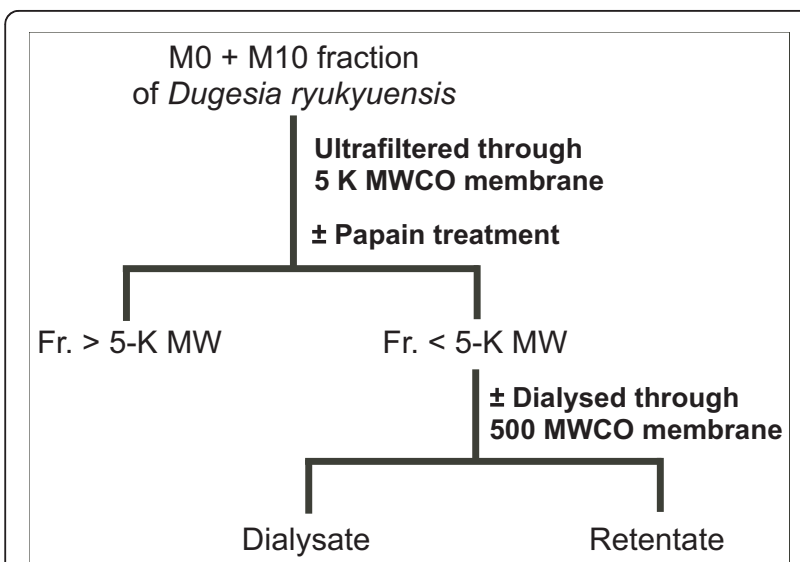

Figure 6 Procedure for fractionation of the M0 + M10 fraction from Dugesia ryukyuensis. See the text for experimental details. Fr., fraction. MW, molecular weight. MWCO, molecular weight cut-off. supernumerary ovaries would be induced by an overdose of the ovary-inducing substance.

Previously, we concluded that an action of the sexinducing substance induces the production of the sexinducing substance in otherwise asexual worms. Once the worms acquired sexuality, they gained the ability to produce the sex-inducing substance [13]. It was suggested that the head portion of B. brunnea lacked some of the sex-inducing substances necessary for the complete sexual induction of asexual worms, whereas the portions other than the head contained sufficient amounts of the sex-inducing substance [8]. Following Sakurai [8], the test worms with acquired sexuality were cut into 3 pieces, and the 3 fragments were allowed to regenerate. The head $(\mathrm{H})$ fragments regenerated to become asexual, whereas the middle $(\mathrm{M})$ and tail $(\mathrm{T})$ fragments regenerated to become sexual [[13], Additional file 3]. In this study, we fed the test worms the $\mathrm{M} 0+\mathrm{M} 10$ fractions derived from the 3 fragments of conspecific sexual worms (Table 5). The activity of the sex-inducing substance in the M0 + M10 fraction was recognised in the $\mathrm{M}$ and $\mathrm{T}$ fragments but not in the $\mathrm{H}$ fragments. These results strongly suggested that the sexinducing substance in the M0 + M10 fraction is involved in the maintenance of acquired sexuality.

The test worms became fully sexual by being fed the < $5-\mathrm{K} \mathrm{MW}$ fraction after protein digestion with papain of the M0 + M10 fraction (Table 6). The putative MW of this sex-inducing substance was estimated at less than 500 by the dialysis experiment (Figure 7 and Table 6). Thus, the sex-inducing substance contained in the M0 + M10 fraction is probably not a protein, although we cannot completely deny that the sex-inducing substance is a protein or a peptide lacking papain-recognition sites. We have also found that the $>5-\mathrm{K} \mathrm{MW}$ fraction clearly enhanced the sex-inducing activity of the $<5-\mathrm{K} \mathrm{MW}$ fraction, although the $>5-\mathrm{K} \mathrm{MW}$ fraction did not have any inherent sex-inducing activity (Figure 7 and Table 6). This indicates that the $>5-\mathrm{K}$ MW fraction probably contains enhancers for the sex-inducing activity of the $<5-\mathrm{K}$ MW fraction. Interestingly, papain treatment of the M0 + M10 fraction seemed to enhance the sex-inducing activity of the $<5-\mathrm{K}$ MW fraction (Figure 7 ).

The sex-inducing substance might contribute to an understanding of the mechanisms underlying the switch 
Table 6 Molecular weight estimation and papain susceptibility testing for a sex-inducing substance

\begin{tabular}{|c|c|c|}
\hline Test food & $\begin{array}{c}\text { Number of test worms that developed a pair } \\
\text { of ovaries (\%) }\end{array}$ & $\begin{array}{c}\text { Number of test worms that developed a } \\
\text { copulatory apparatus (\%) }\end{array}$ \\
\hline Fr. $>5-K M W+F r .<5-K M W$ & $50 / 50(100.0 \pm 0.0)$ & $32 / 50(64.0 \pm 4.0)$ \\
\hline Fr. $>5-K M W$ & $0 / 50(0.0 \pm 0.0)$ & $0 / 50(0.0 \pm 0.0)$ \\
\hline Fr. $<5-K M W$ & $49 / 50(98.0 \pm 2.0)$ & $28 / 50(56.0 \pm 4.0)$ \\
\hline Fr. $<5-K M W$ after papain treatment & $47 / 50(94.0 \pm 6.0)$ & $32 / 50(64.0 \pm 4.0)$ \\
\hline $\begin{array}{l}\text { Retentate after dialysis of papain-treated } \\
\qquad \mathrm{Fr} .<5-\mathrm{K} \mathrm{MW}\end{array}$ & $4 / 50(8.0 \pm 8.0)$ & $0 / 50(0.0 \pm 0.0)$ \\
\hline Control of dialysis experiment & $50 / 50(100.0 \pm 0.0)$ & $27 / 50(54.0 \pm 2.0)$ \\
\hline
\end{tabular}

The M0 + M10 fraction of Dugesia ryukyuensis was obtained as described in Figure 6. See Materials and methods for test food. Control of dialysis experiment: the $<5-\mathrm{K}$ molecular weight (MW) fraction after papain treatment was placed under the same conditions except for dialysis. Means and standard errors of duplicated experiments are shown. Fr., fraction.

from an asexual to a sexual mode of reproduction in planarians. In the multicellular green flagellate Volvox carteri, heat shock elicits the production of a sexual inducer, a 20-kD glycoprotein [16-18]. This protein causes their asexual reproductive cells, called gonidia, to undergo a modified pattern of embryonic development, resulting in the production of gametes, depending on the genetic sex of the individual [19]. However, such compounds have not been reported in metazoans. We

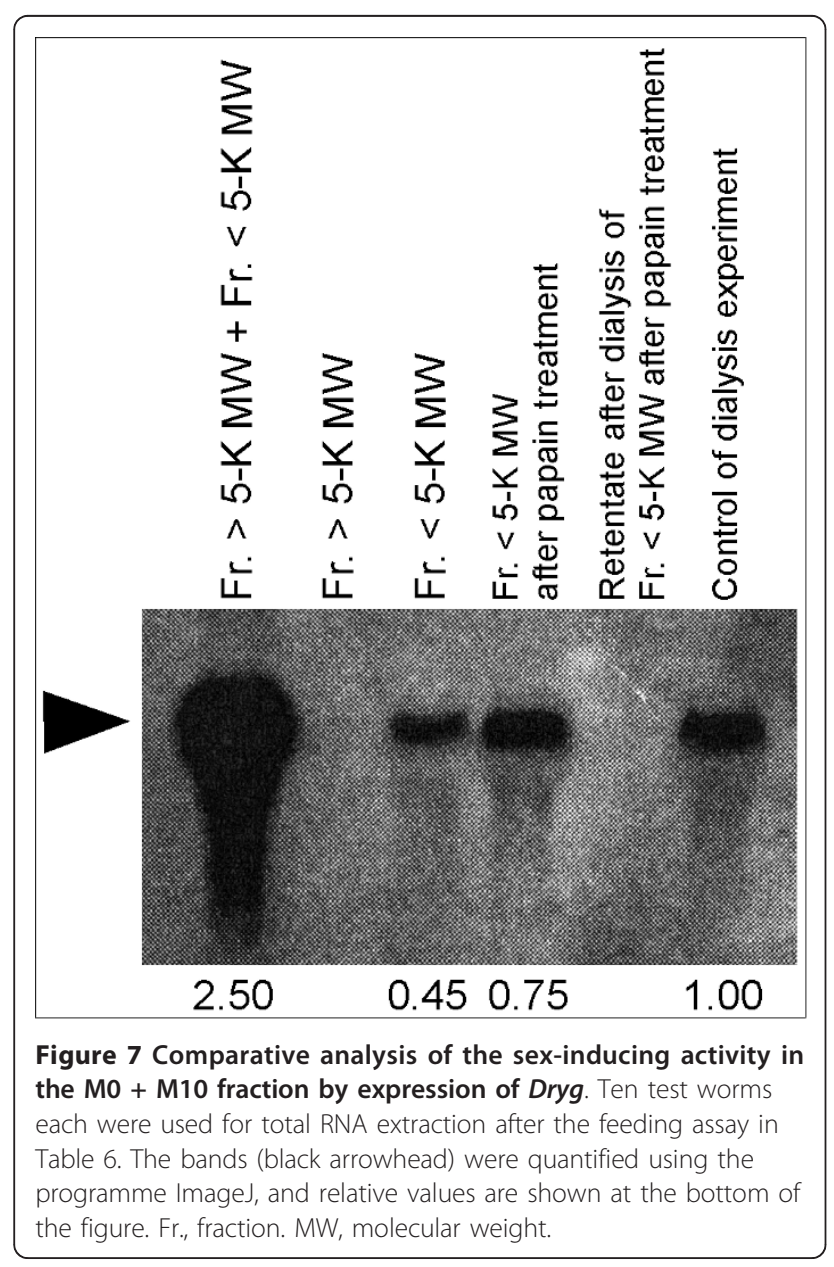

suggest that one of the planarian sex-inducing substances could be a hydrophilic compound with a low MW, i.e. less than 500. Sexual planarians seem to contain steroids such as testosterone and estradiol $[20,21]$. Although these steroids are low-MW compounds, they are rather hydrophobic. Indeed, the test worms did not exhibit any sign of sexual induction when fed these steroids (from $1 \mathrm{ng}$ (approximately $1.43 \mathrm{pg} \cdot \mathrm{day}^{-1} \cdot \mathrm{worm}^{-1}$ ) to $1 \mu \mathrm{g}$ (approximately $\left.1.43 \mathrm{ng} \cdot \mathrm{day}^{-1} \cdot \mathrm{worm}^{-1}\right)$ ) under the bioassay conditions in this study. Thus, these steroids are not the sex-inducing substance. Recently, it has been reported that a neuropeptide Y (NPY) superfamily member plays an important role in the normal development and maintenance of the planarian reproductive system in Schmidtea mediterranea [22]. Particularly, $n p y-8$ is expressed in sexual but not asexual worms. RNAi treatment for $n p y-8$ facilitated regression of the reproductive organs. The predicted peptide size of NPYs is approximately 30 amino acid residues (approximately $3 \mathrm{~K} \mathrm{MW}$ ) $[23,24]$. In terms of MW, NPYs are inconsistent with our putative sex-inducing substance. If the sex-inducing substance is a peptide, it might be another neuropeptide of about 2-4 amino acid residues.

Sex-inducing activity in the M0 and M10 fractions was increased only about 3 - and 7 -fold, respectively (Table 3). Probably, the precipitates contain a large amount of the hydrophobic sex-inducing substance (Figure 1 and Table 1). Sexual induction might be triggered by additive or synergistic effects of the hydrophobic and the hydrophilic sex-inducing substance, including the enhancer. Thus, in terms of purification of the hydrophilic sex-inducing substance, the procedure established is useful. In the near future, we expect that a sex-inducing substance will be isolated by HPLC purification in the bioassay system outlined in the present study and identified by NMR analysis.

\section{Conclusions}

We greatly improved the purification procedure of a planarian sex-inducing substance with our bioassay system. We showed that sex-inducing activity was 
recovered in the cytosolic fraction as well as in the precipitate. We clearly showed that the sex-inducing substance in the cytosolic fraction is hydrophilic, papainresistant, and a putative low-MW compound of less than 500 . We also suggested the presence of an enhancer of sexual induction in the hydrophilic fraction. We conclude that sexual induction might be triggered by additive or synergistic effects of the hydrophobic sex-inducing substance in the precipitate and the sex-inducing substance and its enhancer in the hydrophilic fraction.

\section{Materials and methods}

Animals

An exclusively asexual strain, the $\mathrm{OH}$ strain, of the planarian $D$. ryukyuensis $[25,26]$ was maintained at $20^{\circ} \mathrm{C}$ in dechlorinated tap water and fed chicken liver once a week. The worms of this strain were used as test animals for sexual induction. Wild populations of B. brun$n e a$, an oviparous planarian, were collected in the vicinity of Yamagata City, Japan, and then frozen in liquid nitrogen and stored at $-80^{\circ} \mathrm{C}$. The sexual worms of $D$. ryukyuensis were obtained by feeding the worms of the $\mathrm{OH}$ strain with B. brunnea [27], as described previously [10]. The sexual worms of D. ryukyuensis were cut and allowed to regenerate. They were maintained at $20^{\circ} \mathrm{C}$ in dechlorinated tap water and fed chicken liver once a week, until maturity. Then, they were stored at $-80^{\circ} \mathrm{C}$ as a source of the sex-inducing substance.

\section{Preparation of the extracts from sexual worms}

Approximately 320 intact worms of D. ryukyuensis (4 g in wet weight) and approximately 65 intact worms of $B$. brunnea (4 $\mathrm{g}$ in wet weight) were frozen in liquid nitrogen and kept at $-80^{\circ} \mathrm{C}$ until use. Additionally, sexual worms of $D$. ryukyuensis were cut into 3 pieces with a razor. By surgical ablation, 3 different fragments were obtained with respect to the topological position of the sexual organs: the $\mathrm{H}$ fragment had no sexual organs; the $\mathrm{M}$ fragment had a pair of ovaries, testes, and yolk glands; and the $\mathrm{T}$ fragment had testes, yolk glands, and the copulatory apparatus (Figure 5). Pooled frozen fragments ( $4 \mathrm{~g}$ in wet weight; $\mathrm{H}$ fragments from approximately 2000 worms, and $\mathrm{M}$ and $\mathrm{T}$ fragments from approximately 600 worms) were prepared. Pooled frozen samples were homogenised in $240 \mathrm{~mL}$ of PBS $(34 \mathrm{mM}$ $\mathrm{NaCl}, 0.68 \mathrm{mM} \mathrm{KCl}, 2.5 \mathrm{mM} \mathrm{Na}_{2} \mathrm{HPO}_{4}$, and $0.45 \mathrm{mM}$ $\mathrm{KH}_{2} \mathrm{PO}_{4} ; \mathrm{pH}$ 7.4). The homogenate was centrifuged at $16000 \times g$ for $30 \mathrm{~min}$ at $4^{\circ} \mathrm{C}$. The precipitates were stored at $-80^{\circ} \mathrm{C}$ until the bioassay. The supernatants were filtrated using a $0.2 \mu \mathrm{m}$ filter (CORNING, Lowell, $\mathrm{MA})$ and then centrifuged at $120000 \times g$ for $1 \mathrm{~h}$ at $4^{\circ} \mathrm{C}$. The precipitates were stored at $-80^{\circ} \mathrm{C}$ until the bioassay. The supernatants were loaded onto a Sep-Pak ${ }^{\circledR}$ Light $\mathrm{tC}_{18}$ Cartridge (Waters, Milford, MA) and eluted with
$0 \%, 10 \%, 30 \%$, and $100 \%$ aqueous methanol and hexane to give the following fractions: M0, M10, M30, M100, and $\mathrm{H}$, respectively (Figure 1 ). Each fraction was dried, mixed with $150 \mu \mathrm{l}$ of chicken liver homogenate, freezedried, and used as test food for sexual induction. Twenty-five test worms were fed daily on a piece of food over 4 weeks in standard experiments.

\section{Estimation of sex-inducing activity}

To evaluate the degree of sexual induction in the test worms, weekly external observations were carried out under a binocular microscope, specifically paying attention to the development of a pair of ovaries and a copulatory apparatus. Furthermore, we examined the expression for Dryg by northern blotting. The total RNA of the test worms was prepared using the guanidinum isothiocyanate/phenol-chloroform method [28]. To analyse the RNA fragments by northern blotting [29], we separated $7.5 \mu \mathrm{g}$ of total RNA on a 1\% agarose gel containing formaldehyde, and transferred them to a positively charged nylon membrane. Antisense $\mathrm{P}^{32}$-labelled cDNA probes were prepared with a random prime-labelling system (Amersham Pharmacia Biotech, Schenectady, NY). Hybridisation was carried out at $42^{\circ} \mathrm{C}$ for $16 \mathrm{~h}$ in the hybridisation solution ( $4 \times$ standard sodium citrate [SSC], $50 \%[\mathrm{v} / \mathrm{v}$ ] formamide, $0.2 \%[\mathrm{w} / \mathrm{v}]$ sodium dodecyl sulfate [SDS], $5 \times$ Denhardt's solution, and $0.12 \mathrm{mg} / \mathrm{mL}$ salmon sperm DNA). Post-hybridisation washing was carried out at $50^{\circ} \mathrm{C}$ for $10 \mathrm{~min}$ in $2 \times \mathrm{SSC}-0.1 \% \mathrm{SDS}$. Then, the signals were detected with a BAS 5000 Bio-Image Analyzer (Fuji Photo Film, Japan). The bands from the northern blots were quantified using the programme ImageJ 1.44.

\section{Papain digestion, ultrafiltration experiment, and dialysis experiment for the M0 + M10 fraction}

The M0 + M10 fraction was obtained as shown in Figure 1 and separated as shown in Figure 6. It was digested at $37^{\circ} \mathrm{C}$ for $12 \mathrm{~h}$ in $10 \mu \mathrm{g} / \mathrm{mL}$ of papain (SIGMA-ALDRICH, St. Louis, MO) in 1/10 PBS, after which it was ultrafiltrated using a Vivaspin 20-5 K (GE Healthcare, Little Chalfont, UK). The $<5 \mathrm{~K}$ molecular weight (MW) fraction was dialysed at $4^{\circ} \mathrm{C}$ for $48 \mathrm{~h}$ using 500 molecular weight cut-off (MWCO) dialysis membranes (Spectra/Por ${ }^{\circledR}$ Biotech CE Membrane; SPECTRUM, Rancho Dominguez, CA). Each sample was freeze-dried, mixed with $120 \mu \mathrm{l}$ of chicken liver homogenate, freeze-dried again, and used as test food for sexual induction. Twenty-five test worms were fed daily on a piece of food over 3 weeks.

\section{Statistics}

The sample size in the feeding treatments with the M0 + M10 fraction was 25 . The induction of supernumerary ovary pairs was evaluated using the Welch's $t$-test. 


\section{Additional material}

\section{Additional file 1: Illustration of 5 distinct stages along with the} sexual induction. The development and topological position of reproductive organs are shown. Coloured regions correspond with the sexual organs: red, ovary; blue, testis; yellow, yolk gland; green, a copulatory apparatus with a genital pore. The white region in the body is the pharynx. Briefly, in stage 1, the ovaries became sufficiently large to be externally apparent behind the head, yet no oocytes or other sexual organs were detectable. In stage 2, oocytes appeared in the ovaries, but other sexual organs remained undetectable. In stage 3, the primordial testes emerged and a copulatory apparatus became visible as a white speck in the post-pharyngeal region. In stage 4, yolk gland primordia developed, and spermatocytes appeared in the testes. In stage 5, matured yolk glands formed, and many matured spermatozoa were detectable in the testes. Figure 1 in Kobayashi and Hoshi [12] was modified.

\section{Additional file 2: Induction of supernumerary ovary pairs by} feeding with Bdellocephala brunnea. Asexual worms of Dugesia japonica became fully sexual and induced several supernumerary ovary pairs along the ventral nerve cord and reaching to the pharyngeal level, when they were fed Bdellocephala brunnea, an oviparous species [8]. In general, it is believed that sexual planarians (triclads) have only a pair of ovaries behind the head. Usually, sexual worms of D. ryukyuensis also have a pair of ovaries when fed chicken liver daily (a laboratory condition). However, sometimes some worms develop a pair of supernumerary ovaries in the condition. Additionally, we collected a few sexual worms of $D$. ryukyuensis with a few supernumerary ovary pairs in a natural habitat (lat $26^{\circ} 34^{\prime} 01.76^{\prime \prime} \mathrm{N}$, long $128^{\circ} 02^{\prime} 14.74^{\prime \prime} \mathrm{E}$ : Oura river, Okinawa Prefecture, Japan). Some Dugesia worms might have the ability to produce supernumerary ovary pairs spontaneously. We showed that in the test worms with acquired sexuality, many supernumerary ovary pairs were apparently induced by feeding them with B. brunnea. (A) Test worms with acquired sexuality induced significantly more supernumerary ovary pairs by feeding with $B$. brunnea than that with chicken liver for 2 weeks (Welch's $t$-test: $n_{\text {Minced B. brunnea }}=15, n_{\text {Liver }}=15 ; P=8.44 \mathrm{E}-06$ ). Error bars represent the standard error. (B-D) Morphological examination of test worms with supernumerary ovary pairs. Ventral view of a test worm with acquired sexuality fed chicken liver (B) and B. brunnea (C). Scale bar: $2 \mathrm{~mm}$. Sagittal section of a test worm with supernumerary ovary pairs (D). Scale bar: $500 \mu \mathrm{m}$. Arrowheads represent a main ovary (black) and a supernumerary ovary (red); t, testes; y, yolk glands. The images are arranged with the anterior side at the top.

Additional file 3: Reproductive mode after surgical ablation in the test worms with acquired sexuality. Fifty-six test worms with acquired sexuality were cut as described previously [[13], Figure 5]. Fragments were transferred separately to a plastic dish (diameter, $3 \mathrm{~cm}$ ) containing dechlorinated tap water, allowed to regenerate there at $20^{\circ} \mathrm{C}$, and fed chicken liver once a week. External observations were performed weekly to determine whether regenerated worms became sexual or asexual.

\section{Acknowledgements}

We thank Dr. Sachiko Ishida, Hirosaki University, for her kind gift of the $\mathrm{OH}$ strain. We also thank Dr. Makoto Kitamura of our group and Dr. Hiroshi Hirota, RIKEN Chemical Biology Core Facility for their fruitful comments. Further, we would like to thank Dr. Yuni Nakauchi and Mr. Ryo Sasaki for their invaluable assistance in collecting animals. This work was supported in part by a Grant-in-Aid for Scientific Research (Nos. 15770147 and 20116007) from the Ministry of Science, Culture, Sports and Education, Japan (KK), and from PRESTO, Japan Science and Technology Corporation (KK).

\section{Author details}

${ }^{1}$ Center for Integrated Medical Research, School of Medicine, Keio University, 35 Shinjuku-ku, Tokyo, 160-8582, Japan. ${ }^{2}$ The Open University of Japan, 2-11 Wakaba, Mihama-ku, Chiba, 261-8586, Japan.

\section{Authors' contributions}

The experiment was designed and discussed by KK and MH. Data acquisition, analysis, and drafting of the manuscript were done by KK, with critical revision and major improvements by $\mathrm{MH}$. All authors read and approved the final manuscript.

\section{Competing interests}

The authors declare that they have no competing interests.

Received: 9 July 2011 Accepted: 17 October 2011

Published: 17 October 2011

\section{References}

1. Curtis WC: The life history, the normal fission and the reproductive organs of Planaria maculata. Proc Boston Soc Nat Hist 1902, 30:515-559.

2. Hyman LH: North American triclad Turbellaria. IX. The priority of Dugesia Girard 1850 over Euplanaria Hesse 1897 with notes on American species of Dugesia. Trans Am Micros Soc 1939, 58:264-275.

3. Vowinckel $C$ : The role of illumination and temperature in the control of sexual reproduction in the planarian Dugesia tigrina (Girard). Biol Bull 1970, 138:77-87.

4. Vowinckel C, Marsden JR: Reproduction of Dugesia tigrina under shortday and long-day conditions at different temperatures. I. Sexually derived individuals. J Embryol Exp Morphol 1971, 26:587-598.

5. Vowinckel C, Marsden JR: Reproduction of Dugesia tigrina under shortday and long-day conditions at different temperatures. II. Asexually derived individuals. J Embryol Exp Morphol 1971, 26:599-609.

6. Kenk R: Sexual and asexual reproduction in Euplanaria tigrina (Girard). Biol Bull 1937, 73:280-294.

7. Grasso M, Benazzi M: Genetic and physiologic control of fissioning and sexuality in planarians. J Embryol Exp Morphol 1973, 30:317-328.

8. Sakurai T: Sexual induction by feeding in an asexual strain of the freshwater planarian, Dugesia japonica japonica. Annot Zool Jap 1981 54:103-112.

9. Grasso M, Montanaro L, Quaglia A: Studies on the role of neurosecretion in the induction of sexuality in a planarian agamic strain. J Ultrastruct Res 1975, 52:404-408.

10. Kobayashi K, Koyanagi R, Matsumoto M, Cabrera JP, Hoshi M: Switching from asexual to sexual reproduction in the planarian Dugesia ryukyuensis: Bioassay system and basic description of sexualizing process. Zoolog Sci 1999, 16:291-298.

11. Hoshi M, Kobayashi K, Arioka S, Hase S, Matsumoto M: Switching from asexual to sexual reproduction in the planarian Dugesia ryukyuensis. Integ Comp Biol 2003, 43:242-246.

12. Kobayashi K, Hoshi M: Switching from asexual to sexual reproduction in the planarian Dugesia ryukyuensis: Change of the fissiparous capacity along with the sexualizing process. Zoolog Sci 2002, 19:661-666.

13. Kobayashi K, Arioka S, Hase S, Hoshi M: Signification of the sexualizing substance produced by the sexualized planarians. Zoolog Sci 2002, 19:667-672.

14. Kobayashi K, Arioka S, Hoshi M: Seasonal changes in the sexualization of the planarian Dugesia ryukyuensis. Zoolog Sci 2002, 19:1267-1278.

15. Hase S, Kobayashi K, Koyanagi R, Hoshi M, Matsumoto M: Transcriptional pattern of a novel gene, expressed specifically after the point-of-noreturn during sexualization, in planaria. Dev Genes Evol 2003, 212:585-592.

16. Starr RC, Jaenicke L: Purification and characterization of the hormone initiating sexual morphogenesis in Volvox carteri f. nagariensis lyengar. Proc Natl Acad Sci USA 1974, 71:1050-1054.

17. Kirk DL, Kirk MM: Heat shock elicits production of sexual inducer in Volvox. Science 1986, 231:51-54.

18. Mages HW, Tschochner H, Sumper M: The sexual inducer of Volvox carteri. Primary structure deduced from c DNA sequence. FEBS Lett 1988, 234:407-410.

19. Sumper M, Berg E, Wenzl S, Godl K: How a sex pheromone might act at a concentration below 10-16 M. EMBO J 1993, 12:831-836.

20. Fukushima M, Funabiki I, Hashizume T, Osada K, Yoshida W, Ishida S: Detection and changes in levels of testosterone during spermatogenesis in the freshwater planarian Bdellocephala brunnea. Zoolog Sci 2008, 25:760-765. 
21. Miyashita H, Nakagawa H, Kobayashi K, Hoshi M, Matsumoto M: Effects of 17ß-estradiol and bisphenol A on the formation of reproductive organs in planarian. Biol Bull 2011, 220:47-56.

22. Collins JJ III, Hou X, Romanova EV, Lambrus BG, Miller CM, Saberi A, Sweedler JV, Newmark PA: Genome-wide analysis reveal a role for peptide hormones in planarian germline development. PLOS Biol 2010, 8:1-21.

23. Colmers WF, Bahh BE: Neuropeptide Y and epilepsy. Epilepsy Curr 2003, 3:53-58.

24. Sperk G, Hamilton T, Colmers WF: Neuropeptide $Y$ in the dentate gyrus. Prog Brain Res 2007, 163:285-297.

25. Kawakatsu M, Oki I, Tamura S, Sugino H: Studies on the morphology, karyology and taxonomy of the Japanese freshwater planarian Dugesia japonica Ichikawa et Kawakatsu, with a description of a new subspecies, Dugesia japonica ryukyuensis subspec. The Bulletin of Fuji Women's College 1976, 23:127-132.

26. Kawakatsu M, Oki I, Tamura S: Taxonomy and geographical distribution of Dugesia japonica and D. ryukyuensis in the Far East. Hydrobiologia 1995, 305:55-61.

27. Ijima I, Kaburaki T: Preliminary descriptions of Japanese Triclads. Annot Zool Jap 1916, 9:153-171.

28. Chomczynski P, Sacchi N: Single-step method of RNA isolation by thiocyanate- phenol-chloroform extraction. Anal Biochem 1987, 162:156-159.

29. Alwine JC, Kemp DJ, Stark GR: Method for detection of specific RNAs in agarose gels by transfer to diazobenzyloxymethyl-paper and hybridization with DNA probes. Proc Natl Acad Sci USA 1997, 74:5350-5354.

doi:10.1186/1742-9994-8-23

Cite this article as: Kobayashi and Hoshi: Sex-inducing effect of a hydrophilic fraction on reproductive switching in the planarian Dugesia ryukyuensis (Seriata, Tricladida). Frontiers in Zoology 2011 8:23.

\section{Submit your next manuscript to BioMed Central and take full advantage of:}

- Convenient online submission

- Thorough peer review

- No space constraints or color figure charges

- Immediate publication on acceptance

- Inclusion in PubMed, CAS, Scopus and Google Scholar

- Research which is freely available for redistribution

Submit your manuscript at www.biomedcentral.com/submit
C Biomed Central 